The Astrophysical Journal, 671:1256-1263, 2007 December 20

(C) 2007. The American Astronomical Society. All rights reserved. Printed in U.S.A.

\title{
BLACK HOLE MASS AND GROWTH RATE AT HIGH REDSHIFT
}

\author{
Hagai Netzer, ${ }^{1}$ Paulina Lira, ${ }^{2}$ Benny Trakhtenbrot, ${ }^{1}$ Ohad Shemmer, ${ }^{3}$ and Iara Cury ${ }^{4}$ \\ Received 2007 May 17; accepted 2007 August 27
}

\begin{abstract}
We present new $H$ - and $K$-band spectroscopy of 15 high-luminosity active galactic nuclei (AGNs) at redshifts 2.3-3.4 obtained with Gemini South. We combined the data with spectra of an additional 29 high-luminosity sources to obtain a sample with $10^{45.2}<\lambda L_{\lambda}(5100 \AA)<10^{47.3} \mathrm{ergs} \mathrm{s}^{-1}$ and a black hole $(\mathrm{BH})$ mass range, using reverberation mapping relationships based on the $\mathrm{H} \beta$ method, of $10^{8.8}-10^{10.7} M_{\odot}$. We do not find a correlation of $L / L_{\mathrm{Edd}}$ with $M_{\mathrm{BH}}$, but we do find a correlation with $\lambda L_{\lambda}(5100 \AA)$, which might be due to selection effects. The $L / L_{\text {Edd }}$ distribution is broad and covers the range of $\sim 0.07-1.6$, similar to what is observed in lower redshift, lower luminosity AGNs. We suggest that this consistently measured and calibrated sample gives the best representation of $L / L_{\text {Edd }}$ at those redshifts, and note potential discrepancies with recent theoretical and observational studies. The lower accretion rates are not in accord with growth scenarios for BHs at such redshifts, and the growth times of many of the sources are longer than the age of the universe at the corresponding epochs. This suggests earlier episodes of faster growth at $z \gtrsim 3$ for those sources. The use of the $\mathrm{C}$ IV $\lambda 1549$ method gives considerably different results and a larger scatter; this method seems to be a poor $M_{\mathrm{BH}}$ and $L / L_{\text {Edd }}$ estimator at very high luminosity.
\end{abstract}

Subject headings: galaxies: active — galaxies: nuclei — quasars: emission lines

\section{INTRODUCTION}

Studies of black hole $(\mathrm{BH})$ growth at various redshifts, and the comparison with galaxy evolution and star formation, has been a very active area of research for several years. In particular, there have been several suggestions that very massive BHs grew faster at early epochs, while the growth of less massive BHs extends over longer periods and is significant even at $z=0$. For example, Marconi et al. (2004) used the X-ray luminosity function of active galactic nuclei (AGNs) to suggest a specific growth pattern as a function of cosmic time. According to these authors, BHs with $M_{\mathrm{BH}} \gtrsim 10^{8} M_{\odot}$ attained $50 \%$ of their mass by $z=2$ and $90 \%$ of their mass by $z=1$ (Marconi et al. 2004, their Fig. 2). Smaller BHs grew slower at earlier times, and many active BHs with $M_{\mathrm{BH}} \sim 10^{7} M_{\odot}$ are still growing today. Similar scenarios, under the general terminology of "antihierarchical growth of supermassive BHs," have been presented by Merloni (2004) and others. Those studies assume that the growth rate of very massive BHs at high redshifts approached the Eddington limit. More recent studies, e.g., by Volonteri et al. (2006) and Hopkins et al. (2006), focus on the importance of the Eddington ratio, $L / L_{\mathrm{Edd}}$, in determining $\mathrm{BH}$ evolution at all redshifts. This also includes hierarchical models for the evolution of the most massive BHs. All these models can be tested observationally by direct measurements of $\mathrm{BH}$ mass and accretion rate at high redshifts, provided high-quality observations and reliable methods for determining $M_{\mathrm{BH}}$ are available.

Current $\mathrm{BH}$ mass estimates are based on reverberation mapping, which provides a way to measure the emissivity-weighted size of the broad-line region (BLR) in type-I AGNs as a function of the optical continuum luminosity $\left(\lambda L_{\lambda}\right.$ at $5100 \AA$, hereafter

\footnotetext{
1 School of Physics and Astronomy and the Wise Observatory, The Raymond and Beverly Sackler Faculty of Exact Sciences, Tel-Aviv University, Tel-Aviv 69978, Israel.

2 Departamento de Astronomía, Universidad de Chile, Camino del Observatorio 1515, Santiago, Chile.

3 Department of Astronomy and Astrophysics, 525 Davey Laboratory, Pennsylvania State University, University Park, PA 16802.

4 Astronomy Department Yale University, P.O. Box 208101, New Haven, CT 06520-8101.
}

$L_{5100}$; see Kaspi et al. 2000, 2005, hereafter K05, and references therein). This scaling has been used to obtain a "single-epoch" estimate of the $\mathrm{BH}$ mass by combining the BLR size derived from $L_{5100}$ with a measure of the gas velocity obtained from $\operatorname{FWHM}(\mathrm{H} \beta)$ (hereafter "the $\mathrm{H} \beta$ method"). There are obvious limitations to this method due to the somewhat vague definition of the BLR size, variable source luminosity, BLR geometry, and somewhat uncertain line widths. There are also questions regarding the exact slope of the BLR size versus the source luminosity (e.g., Bentz et al. 2006). These translate to a factor of $\sim 2$ uncertainty on the derived masses. An additional uncertainty is associated with the limited luminosity range of the K05 sample and hence the need to extrapolate the relationship beyond its highest luminosity end, at $L_{5100} \simeq 2 \times 10^{46} \mathrm{ergs} \mathrm{s}^{-1}$ (see, however, the new result of Kaspi et al. 2007).

Other combinations of continuum luminosity and line widths have also been used, especially in the study of high-redshift sources (e.g., McLure \& Dunlop 2004; Vestergaard 2004; Vestergaard \& Peterson 2006). These seem to be associated with a larger uncertainty on the derived masses, especially when the $\mathrm{C}$ IV $\lambda 1549$ line width, in combination with $\lambda L_{\lambda}$ at $\sim 1400 \AA$ (hereafter "the C IV $\lambda 1549$ method") are used (e.g., Baskin \& Laor 2005; Sulentic et al. 2007). One way to extend the $\mathrm{H} \beta$ method to high redshifts is by high-quality near-infrared spectroscopy. Measurements in a moderate-size sample (29 sources) of this type are presented in Shemmer et al. (2004, hereafter S04), who used $H$ - and $K$-band spectroscopy for obtaining single-epoch $\mathrm{BH}$ masses and accretion rates in very high luminosity AGNs. More recent work of this type (a sample of 17 objects with $1<z<2$ and 4 objects with $z>2$ ) is described in Sulentic et al. (2006).

This paper presents $\mathrm{BH}$ masses and accretion rates obtained with the $\mathrm{H} \beta$ method for a new sample of 15 sources at two redshift bands around $z \simeq 2.3$ and 3.4. Together with our earlier work (S04), we can now use the $\mathrm{H} \beta$ method to investigate the mass and accretion rate of high-redshift sources over a range of 2.5 dex in luminosity. In $\S 2$ we describe the observations and their analysis, and in $\S 3$ we present the main results and a discussion of our findings including an assessment of the growth 
TABLE 1

GNIRS OBSERVATION LOG

\begin{tabular}{|c|c|c|c|c|c|}
\hline $\begin{array}{l}\text { Object ID } \\
\text { (SDSS J) }\end{array}$ & $z_{\text {sys }}{ }^{\mathrm{a}}$ & $z_{\text {SDSS }}{ }^{\mathrm{b}}$ & Band & Observation Date & $\begin{array}{c}\text { Exposure Time } \\
(\mathrm{hr})\end{array}$ \\
\hline $025438.37+002132.8 \ldots \ldots \ldots \ldots \ldots \ldots \ldots \ldots \ldots$ & 2.456 & 2.463 & $H$ & 2005 Dec 7 & 1.0 \\
\hline $083630.55+062044.8 \ldots \ldots \ldots \ldots \ldots \ldots \ldots \ldots \ldots$ & 3.397 & 3.397 & $K$ & 2005 Nov 27 & 1.0 \\
\hline 095141.33+013259.5 ……..................... & 2.411 & 2.429 & $H$ & 2006 Jan 24 & 2.5 \\
\hline $100710.70+042119.1$ & 2.363 & 2.363 & $H$ & 2006 Mar 26 & 2.0 \\
\hline $101257.52+025933.2 \ldots \ldots \ldots \ldots \ldots \ldots \ldots$ & 2.434 & 2.434 & $H$ & $2006 \mathrm{Feb} 08$ & 0.5 \\
\hline 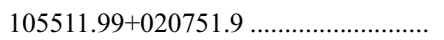 & 3.391 & 3.384 & $K$ & 2005 Apr 21 & 1.0 \\
\hline 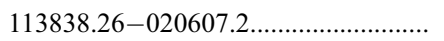 & 3.352 & 3.343 & $K$ & 2005 Apr 21 & 1.0 \\
\hline 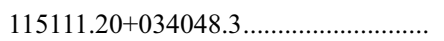 & 2.337 & 2.337 & $H$ & 2006 Apr 16, 25 & 2.5 \\
\hline $115304.62+035951.5$ & 3.426 & 3.432 & $K$ & 2005 Apr 23 & 0.7 \\
\hline $115935.64+042420.0$ & 3.451 & 3.448 & K & 2005 Apr 23 & 0.7 \\
\hline $125034.41-010510.5$ & 2.397 & 2.397 & $H$ & 2006 Apr 26, 27 & 2.7 \\
\hline $144245.66-024250.1$.................................. & 2.356 & 2.343 & $H$ & 2006 Jul 19 & 1.0 \\
\hline 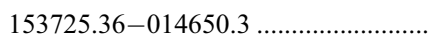 & 3.452 & 3.452 & $K$ & 2005 Apr 21 & 0.7 \\
\hline $210258.22+002023.4 \ldots \ldots \ldots \ldots \ldots \ldots \ldots \ldots \ldots \ldots$ & 3.328 & 3.343 & K & $2006 \mathrm{Jul} 19$ & 1.3 \\
\hline $210311.68-060059.4 \ldots \ldots \ldots \ldots \ldots \ldots \ldots \ldots \ldots \ldots$ & 3.336 & 3.336 & K & 2005 May 31 & 0.7 \\
\hline
\end{tabular}

a Systemic redshift measured from the [O III] lines.

${ }^{b}$ Redshift obtained from the SDSS archive based on rest-frame UV emission lines.

rate of high-redshift AGNs, and the evaluation of BH mass measurements obtained with the $C$ IV $\lambda 1549$ method as a replacement for the $\mathrm{H} \beta$ method at high redshifts.

\section{OBSERVATIONS, DATA REDUCTION, AND MASS DETERMINATION}

The sample described in this paper contains 15 high-redshift, high-luminosity AGNs that were selected by their luminosity and redshift. The redshift range stems from the requirement to directly measure the $\mathrm{H} \beta$ line and the $5100 \AA$ continuum from the ground in order to use the $\mathrm{H} \beta$ method, which we consider to provide the most reliable $\mathrm{BH}$ mass estimates. The redshift ranges are dictated by the wavelengths of the $H$ and $K$ bands and are $\sim 2.1-2.5$ and $\sim 3.2-3.5$, respectively. The luminosity is dictated by the goal of going down the AGN luminosity function, starting from its top, and measuring masses and accretion rates of fainter and fainter sources. Given this, we chose sources that are 510 times less luminous than the mean luminosity in the S04 sample. For the basic sample we chose the Sloan Digital Sky Survey (SDSS; e.g., York et al. 2000), which contains flux-calibrated data and hence luminosity estimates for all sources.

Spectroscopic observations were obtained with the Gemini Near-Infrared Spectrograph (GNIRS) on Gemini South under programs GS-2005B-Q-28, GS-2006A-Q-58, and GS-2005AQ-51. The long-slit observations were acquired in the $H$ or $K$ bands, depending on the redshift of the source. The slit width was $1^{\prime \prime}$, and the targets were nodded along the slit to obtain a good background subtraction. The $32 \mathrm{~mm}^{-1}$ grating was used in all observations, resulting in $R \sim 640$ and $850, \Delta \lambda \sim 1.46-1.89 \mu \mathrm{m}$ and $\Delta \lambda \sim 1.83-2.49 \mu \mathrm{m}$ for the $H$ and $K$ bands, respectively. Typical exposure times of the subintegrations were 400-600 s. More details are given in Table 1, where we list all 15 sources and assign to each the systemic redshift measured from their [O III] $\lambda 5007$ lines. These can be somewhat different from the SDSS redshifts, which were obtained by measuring rest-frame ultraviolet (UV) broad-emission lines and are also listed in the table.

The reduction of the raw spectroscopic data was done using the GEMINI package in IRAF. The pipeline combines observations from different nodded positions to obtain background-subtracted images, determines the wavelength calibration, registers the frames, and produces a final averaged image. Extraction of the spectra and flux calibration were performed using standard IRAF tasks. Special care was taken to correct for telluric absorption. This was done by observing early-type stars right before or after the science targets and at similar air masses.

To obtain a more accurate flux calibration of our spectra, $\mathrm{H}$ and $K$-band photometry was obtained for 13 of our sources using the ISPI detector on the Cerro Tololo Inter-American Observatory $4 \mathrm{~m}$ telescope on 2007 February 12 . The data were reduced in the standard way using the XDIMSUN package in IRAF. ${ }^{5}$ The calibration was achieved using Two Micron All Sky Survey $(2 \mathrm{MASS})^{6}$ stars in the $10^{\prime} \times 10^{\prime}$ field of view of the instrument. Only stars with good quality flags in the 2MASS All-Sky Catalog of Point Sources (Cutri et al. 2003) were used, resulting in a $\sim 10 \%$ accuracy. All luminosities listed below are based on these values except for the two sources that were not observed, for which we use the flux from the spectroscopy. The typical rms difference between the two methods is about $15 \%$.

We applied a fitting procedure similar to the one described in Netzer \& Trakhtenbrot (2007, hereafter NT07) to fit all spectra with the various components expected in this range. In short, we fit a linear continuum between rest frames $\sim 4700$ and $\sim 5100 \AA$ and then a five-component Gaussian emission-line model to the continuum-subtracted spectrum: two components for the broad $\mathrm{H} \beta$ line, one for the narrow $\mathrm{H} \beta$ line, and two for the $[\mathrm{O}$ III] $\lambda \lambda 4959$, 5007 lines. This fit serves to obtain a first estimate of $\operatorname{FWHM}(\mathrm{H} \beta)$. We then use the Boroson \& Green (1992) Fe II template, convolved with a single Gaussian with the above FWHM (i.e., the one obtained by the combination of the two broad components), to fit the Fe II emission complex over the range of 4400-4650 $\AA$. This fit, extended to the entire wavelength range by using the template, is used to estimate the Fe II line contributions to the continuum bands and to improve the continuum definition. Using the initial Fe II template, we obtain a modified continuum-subtracted spectrum and repeat the line-fitting process. A second iteration Fe II model is obtained and then subtracted from the spectrum,

\footnotetext{
${ }^{5}$ IRAF (Image Reduction and Analysis Facility) is distributed by the National Optical Astronomy Observatories, which are operated by the Association of Universities for Research in Astronomy, Inc. under a cooperative agreement with the National Science Foundation.

6 See http://www.ipac.caltech.edu/2mass.
} 

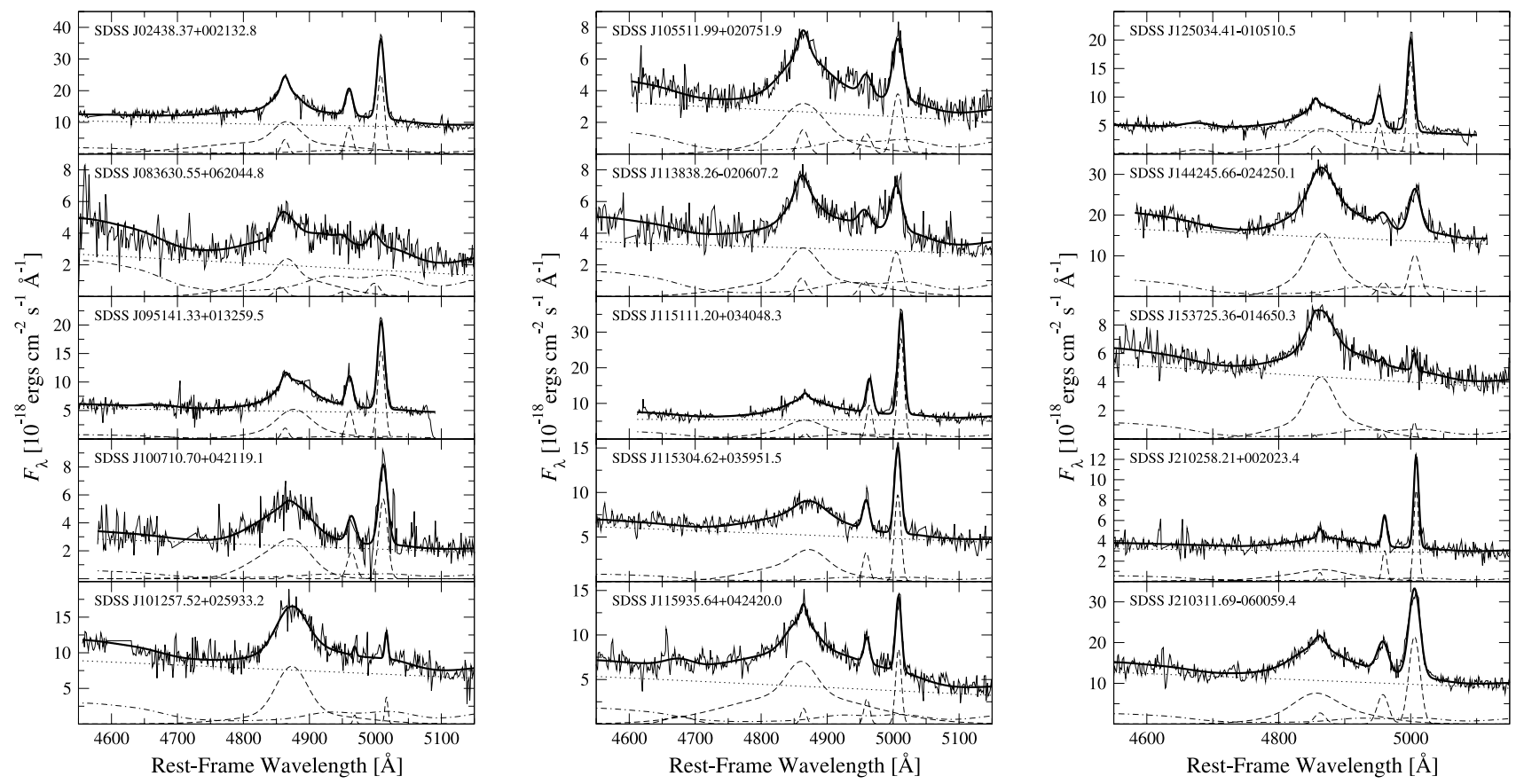

FIG. 1. - GNIRS spectra of our sample of 15 high-redshift AGNs. The best-fit model (thick solid curve) in each panel is composed of a continuum component (dotted line), an Fe II-emission complex (thin dot-dashed curve), and $\mathrm{H} \beta$ and $[\mathrm{O}$ III] emission lines (thick dot-dashed lines).

and a final five-component Gaussian fit of the $\mathrm{H} \beta$ and [O $\mathrm{III}]$ lines is performed. In the final stage the two BLR Gaussians are limited to $1500<\mathrm{FWHM}<20,000 \mathrm{~km} \mathrm{~s}^{-1}$, and the NLR components are forced to have the same FWHM for all three lines. This component is not allowed to exceed $1200 \mathrm{~km} \mathrm{~s}^{-1}$, which is smaller than the FWHM([O III] $]$ values of some of the $\mathrm{S} 04$ sources (see Netzer et al. 2004, their Table 1), but appears to be adequate for the present sample. The monochromatic luminosity at $5100 \AA$, $L_{5100}$, is measured directly from the fitted continuum. The results of our line-fitting procedure are given in Figure 1, where we show the observed spectrum, the model components, and the final fit for all 15 sources.

The measurement of $M_{\mathrm{BH}}$ is a crucial point and requires some discussion. The K05 study suggests that the correlation of BLR size as deduced from the $\mathrm{H} \beta$ lag with respect to the optical continuum is given by $R_{\mathrm{BLR}} \propto L^{\alpha}$, where $\alpha=0.65-0.7$. This slope is obtained from fitting 34 sources covering about 4 orders of magnitude in $L_{5100}$. More recent work by Bentz et al. (2006) used an improved stellar subtraction procedure for several of the lower luminosity sources in the K05 sample, which reduced $L_{5100}$ for those cases. Using 26 sources, they obtained $\alpha=0.52$. A more recent study by the same group (Bentz et al. 2007) that includes four additional sources (but again not the entire K05 sample) with improved stellar subtraction gives $\alpha=0.54 \pm 0.04$.

Given the very large luminosity range (about a factor of $2 \times 10^{4}$ in $L_{5100}$ ) of the K05 sample, it is not at all clear that the slopes at low and high luminosities are the same. It is thus justified to use the higher luminosity sources to obtain the most appropriate slope for extrapolating to luminosities larger than those of the K05 sample. Similarly, we suggest using only the lower luminosity sources in $\mathrm{K} 05$ (after including the Bentz et al. 2007 corrections) when looking for the best extrapolation to very low luminosities. This approach was used by NT07 in their study of the SDSS sample. Since most of the sources analyzed here have extremely large $L_{5100}\left(10^{45.2}-10^{47.3} \mathrm{ergs} \mathrm{s}^{-1}\right)$, we chose to adopt the same approach and obtain a best slope by fitting only those sources in K05 with $L_{5100}>10^{43.5} \mathrm{ergs} \mathrm{s}^{-1}$. This gives the same expression as used by NT07,

$$
M_{\mathrm{BH}}=1.05 \times 10^{8}\left(\frac{L_{5100}}{10^{46} \mathrm{ergs} \mathrm{s}^{-1}}\right)^{0.65}\left[\frac{\mathrm{FWHM}(\mathrm{H} \beta)}{10^{3} \mathrm{~km} \mathrm{~s}^{-1}}\right]^{2} M_{\odot} .
$$

For comparison, we also calculated $M_{\mathrm{BH}}$ and accretion rate using the expression given in Bentz et al. (2007, their eq. [2]). This gives masses that are smaller by a factor of $\sim 1.2$ for our lowest $L_{5100}$ sources, and by a factor of $\sim 1.9$ for our highest luminosity AGNs. These alternative estimates are compared below with the results obtained by using equation (1).

The calculation of $L_{\mathrm{bol}} / L_{\mathrm{Edd}}$ (hereafter $L / L_{\mathrm{Edd}}$ ) is based on a bolometric correction, $f_{L}$, which is somewhat luminosity dependent, and for the sample in hand is of the order 5-7 (NT07, and references therein). For most applications described here we assumed $f_{L}=7$ [i.e., $L / L_{\mathrm{Edd}}=7 L_{5100} /\left(1.5 \times 10^{38} M_{\mathrm{BH}} / M_{\odot}\right)$ ]. Marconi et al. (2004) give a specific expression for $f_{L}$ that translates to bolometric correction factors of 5.4-6.4 for our sample (see also Richards et al. 2006 on this issue, but note the different method of counting the IR luminosity in that paper). We have carried the analysis described below using those values but given the small range in $f_{L}$, the conclusions hardly change. Table 2 shows the results obtained from the above measurements and fits by assuming a standard cosmology with $\Omega_{\Lambda}=0.7, \Omega_{m}=0.3$, and $H_{0}=70 \mathrm{~km} \mathrm{~s}^{-1} \mathrm{Mpc}^{-1}$.

All the 29 spectra of the S04 sample were refitted using a similar procedure, and $\mathrm{BH}$ masses were recalculated using equation (1) (which is slightly different from the one used in S04). Some of the $\mathrm{S} 04$ data are of poorer quality compared to the new GNIRS observations, which resulted in larger uncertainties. In addition, slight mis-centering of the $\mathrm{H} \beta$ line in the $H$ or $K$ bands forced us, in several cases, to perform the $\mathrm{Fe}$ II fit on the $\mathrm{Fe}$ II complex longward of the $\mathrm{H} \beta$ line. Another difference from the S04 procedure 
TABLE 2

Observed and Derived Properties

\begin{tabular}{|c|c|c|c|c|c|c|c|c|}
\hline Object ID (SDSS J) & $z_{\text {sys }}$ & $\begin{array}{l}\log L_{5100} \\
\left(\text { ergs s}^{-1}\right)\end{array}$ & $\begin{array}{c}\log \lambda L_{\lambda}(1450 \AA) \\
\left(\mathrm{ergs} \mathrm{s}^{-1}\right)\end{array}$ & $\begin{array}{c}\operatorname{FWHM}(\mathrm{H} \beta) \\
\left(\mathrm{km} \mathrm{s}^{-1}\right)\end{array}$ & $\begin{array}{c}\text { FWHM(C IV }) \\
\left(\mathrm{km} \mathrm{s}^{-1}\right)\end{array}$ & $\begin{array}{c}\log M_{\mathrm{BH}} \\
\left(M_{\odot}\right)\end{array}$ & $\log L / L_{\mathrm{Edd}}$ & $t_{\text {grow }}{ }^{\mathrm{a}} / t_{\text {universe }}$ \\
\hline $025438.37+002132.8 \ldots \ldots \ldots \ldots \ldots \ldots \ldots$ & 2.456 & 45.85 & 45.93 & 4164 & 4753 & 9.162 & $-0.64 \pm 0.08$ & 0.8 \\
\hline $095141.33+013259.5$ & 2.411 & 45.55 & 45.9 & 4297 & 5289 & 8.997 & $-0.78 \pm 0.15$ & 1.1 \\
\hline $100710.70+042119.1 \ldots \ldots \ldots \ldots \ldots \ldots$ & 2.363 & 45.17 & 45.67 & 5516 & 5495 & 8.96 & $-1.13 \pm 0.15$ & 2.4 \\
\hline 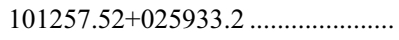 & 2.434 & 45.73 & 45.95 & 3892 & 5862 & 9.029 & $-0.62 \pm 0.08$ & 0.8 \\
\hline $105511.99+020751.9 \ldots \ldots \ldots \ldots \ldots \ldots \ldots$ & 3.391 & 45.70 & 46.24 & 5424 & 5476 & 9.294 & $-0.93 \pm 0.08$ & 2.4 \\
\hline 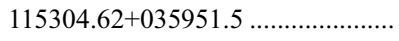 & 3.426 & 46.04 & 46.37 & 5521 & 1773 & 9.529 & $-0.82 \pm 0.08$ & 2.0 \\
\hline $115935.64+042420.0$ & 3.451 & 45.92 & 46.43 & 5557 & 4160 & 9.460 & $-0.89 \pm 0.15$ & 2.2 \\
\hline $125034.41-010510.5 \ldots \ldots \ldots \ldots \ldots \ldots \ldots$ & 2.397 & 45.41 & 45.71 & 5149 & 4234 & 9.061 & $-0.98 \pm 0.08$ & 1.77 \\
\hline $144245.66-024250.1$....................... & 2.356 & 46.03 & 45.90 & 3661 & 3277 & 9.166 & $-0.47 \pm 0.08$ & 0.6 \\
\hline $153725.36-014650.3$.................... & 3.452 & 45.98 & 46.44 & 3656 & 5650 & 9.133 & $-0.49 \pm 0.08$ & 0.9 \\
\hline $210258.22+002023.4$ & 3.328 & 45.79 & 45.86 & 7198 & 2355 & 9.599 & $-1.14 \pm 0.08$ & 4.0 \\
\hline
\end{tabular}

${ }^{\text {a }}$ Assuming $\eta=0.1, f_{L}=7, M_{\text {seed }}=10^{4} M_{\odot}$, and $f_{\text {active }}=1$.

is the inclusion of the narrow $\mathrm{H} \beta$ component in the fits. As a result, several of the newly measured $\operatorname{FWHM}(\mathrm{H} \beta)$ values are somewhat larger than those found by S04. Given all this, we consider the newly fitted $\mathrm{FWHM}(\mathrm{H} \beta)$ values to be more reliable than those presented in S04, although the differences are small, and the main change is the inclusion of an estimate on the uncertainty of the FWHM $(\mathrm{H} \beta)$ measurement (see below). Notable exceptions are six cases (UM 632, 2QZ J231456.8-280102, [HB89] 2254+024, SBS 1425+606, UM 642, and SDSS J024933.42083454.4 ), where the difference in $M_{\mathrm{BH}}$ is of the order $\sim 2-3$ due to the change in the measured $\mathrm{FWHM}(\mathrm{H} \beta)$. The total sample consists of 44 sources covering the luminosity range $10^{45.2}<$ $\lambda L_{\lambda}(5100 \AA)<10^{47.3} \mathrm{ergs} \mathrm{s}^{-1}$. This represents the AGNs luminosity function from about a factor of 2 below the top to a factor of $\sim 200$ below the top.

The main uncertainties on the measured masses are due to uncertainties in $\mathrm{FWHM}(\mathrm{H} \beta)$. To estimate this, we divided all fits into three categories reflecting their quality. Most of the sources show symmetrical lines, and the Fe II complex is easy to model and deconvolve. The assigned uncertainty on $\operatorname{FWHM}(\mathrm{H} \beta)$ in this case is $10 \%$. Some sources have adequate signal-to-noise ratios $(\mathrm{S} / \mathrm{N})$, yet the broad-line profiles are somewhat irregular, and the FWHM is more difficult to constrain. The uncertainty in this case is estimated to be $20 \%$. Finally, in those cases showing asymmetric profiles, difficult-to-model Fe II lines and poorer $\mathrm{S} / \mathrm{N}$, we assigned an uncertainty of $30 \%$ on $\operatorname{FWHM}(\mathrm{H} \beta)$. While this procedure is somewhat subjective, we have no better way to quantify the fitting process. We consider those uncertainties conservative and note that they translate to relative errors on the mass determination of $20 \%-60 \%$. None of the new GNIRS objects is assigned the highest uncertainty of $30 \%$, but six of the S04 sources fall into this category.

We also used the SDSS spectra to measure $\lambda L_{\lambda}(1450 \AA)$ and $\operatorname{FWHM}(\mathrm{C}$ iv 21549$)$ for the 15 new sources. The luminosity is available directly from the observed continuum flux, and the FWHM is obtained by fitting two Gaussians to the line profile. Similar information is available in $\mathrm{S} 04$ for 27 of the 29 sources in their sample.

To summarize, our sample contains almost all sources at $z>2$, where $M_{\mathrm{BH}}$ was obtained using the most robust and reliable method (the $\mathrm{H} \beta$ method). Much larger uncertainties are associated with the $\mathrm{C}$ Iv $\lambda 1549$ method (see detailed discussion below), the one used in almost all other $z>2$ studies. Thus, we believe that our data set is the most suitable and most accurate to address the issues of $\mathrm{BH}$ growth and the distribution of $M_{\mathrm{BH}}$ and $L / L_{\mathrm{Edd}}$ at those redshifts.

\section{DISCUSSION}

\subsection{Luminosity and $L / L_{\mathrm{Edd}}$ in High-Redshift $A G N s$}

We have looked for correlations between $L / L_{\mathrm{Edd}}$ and various other properties of the 44 high-redshift sources in our sample. The correlation with $L_{5100}$ is shown in Figure $2 a$. The two subsamples are shown with different symbols emphasizing the difference in the mean luminosity. Standard regression analysis indicates a significant correlation with a Pearson correlation coefficient of $0.45\left(p=2 \times 10^{-3}\right)$ and a Spearman-rank correlation coefficient of $0.46\left(p=2 \times 10^{-3}\right)$. We also checked the correlation of $L / L_{\mathrm{Edd}}$ with $M_{\mathrm{BH}}$. The diagram is shown in Figure $2 b$ and exhibits a large scatter and no apparent correlation. In particular, the scatter in $L / L_{\text {Edd }}$ (about a factor of 10 ) is similar for BHs of all masses.

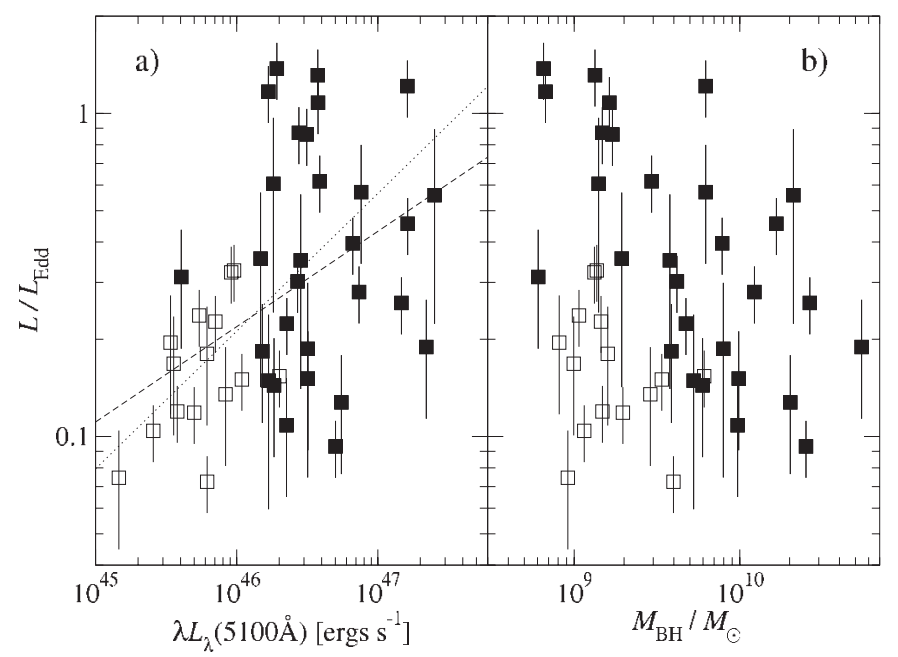

FIG. 2.- (a) $L / L_{\mathrm{Edd}}$ vs. $L_{5100}$ and (b) $M_{\mathrm{BH}}$ for the entire sample of 44 luminous, high-redshift AGNs. GNIRS S04 sources are marked with open and filled symbols, respectively. The dashed and dotted lines mark two best-fit lines obtained with two, somewhat different statistical methods with slopes 0.29 and 0.43 . 
We have repeated the analysis using, this time, the Bentz et al. (2007) expression for estimating $M_{\mathrm{BH}}$. The correlation of $L / L_{\mathrm{Edd}}$ with $L_{5100}$ is even stronger, and again, there is no correlation of $L / L_{\mathrm{Edd}}$ with $M_{\mathrm{BH}}$. As explained, all $M_{\mathrm{BH}}$ values calculated in this way are smaller by factors of $1.2-1.9$, depending on their luminosity. As a result, all values of $L / L_{\text {Edd }}$ are larger by similar factors. This results in some sources with $L / L_{\text {Edd }} \sim 3$. We suspect that the extremely large accretion rates may not be physical, but given the method uncertainty and the extrapolation beyond the K05 luminosity range, we cannot rule them out. On the other hand, the deduced $M_{\mathrm{BH}}$ in this case is smaller, on average by a factor 1.5 , which may be more consistent with the lack of very high mass BHs at low redshifts (but note that even the Bentz et al. relationship gives several cases with $M_{\mathrm{BH}}>10^{10} M_{\odot}$ ).

The slopes of the above correlations depend on the statistical method used and are not too different from the one expected from a case where $\operatorname{FWHM}(\mathrm{H} \beta)$ is independent of $L_{5100}$ (eq. [1]). Indeed, there is no correlation between $\operatorname{FWHM}(\mathrm{H} \beta)$ and $L_{5100}$ in our sample. This point requires some explanation. Single-epoch mass determination provides reliable $\mathrm{BH}$ mass estimates only because of a (yet to be explained) scaling of the BLR size with source luminosity (discovered by reverberation mapping) and the virial motion of the BLR gas. Given this scaling, a complete and unbiased sample must also show some dependence of the mean gas velocity on source luminosity, depending on the distribution of $M_{\mathrm{BH}}$ in the sample. This is not observed in the sample at hand. It may reflect the incompleteness of the sample, its small size, or the real $M_{\mathrm{BH}}$ distribution. Thus, the above correlations are not, by themselves, very important. The more significant finding is the presence of a large number of very massive BHs, at high redshifts, with $L / L_{\text {Edd }}$ considerably smaller than unity. In this respect, there is no difference between the two redshift groups presented here. As discussed below, this is relevant to the question of $\mathrm{BH}$ growth in the early universe.

The recent work of Kollmeier et al. (2006, hereafter K06) includes a systematic study of $\mathrm{BH}$ masses and accretion rates in a sample of 407 AGNs covering the redshift range of 0.3-4. These authors suggest a very narrow range of $L / L_{\text {Edd }}$, at all luminosities and redshifts, consistent with $\log \left(L / L_{\text {Edd }}\right)=-0.6 \pm 0.3$. The range is even smaller ( $0.28 \mathrm{dex})$ for 131 high-luminosity, highredshift $(z>1.2$ by their definition) sources that are more relevant to the present discussion. The paper suggests that the intrinsic distribution in $L / L_{\text {Edd }}$ is even narrower ( 0.24 dex for the high- $z$ high- $L$ subgroup) and much of the observed scatter is due to uncertainties in $\mathrm{BH}$ mass determination and bolometric correction.

Our sample contains a similar number of sources to K06 in the 2.1-3.5 redshift range. Moreover, the number of K06 sources in the redshift and $M_{\mathrm{BH}}$ range of our sample is much smaller (only 19 compared with our 44). Thus, our sample is more suitable, in terms of number of sources, to address the issue of the $L / L_{\mathrm{Edd}}$ distribution in the population of high-redshift, large $\mathrm{BH}$ mass AGNs. We find a broader range in $L / L_{\text {Edd }}$, compared with the various subgroups presented in K06. For example, $90 \%$ of the 44 sources are found in the accretion rate interval $0.08<$ $L / L_{\text {Edd }}<1.5$. The source of the difference between our results and those of K06 is not entirely clear. It may be related to the large uncertainty, and perhaps even a bias, in the method they used to determine $M_{\mathrm{BH}}$ (most masses in their high- $z$ high- $L$ group and all masses for $z>2$ sources were determined with the C IV $\lambda 1549$ method; see also comment on $L / L_{\text {Edd }}$ measured this way in $\S$ 3.4). The K06 sample is flux limited and thus more complete than ours. However, as explained, the number of sources in individual mass and redshift bins are extremely small. These conclusions do not change when using the Bentz et al. (2007) $M_{\mathrm{BH}}$

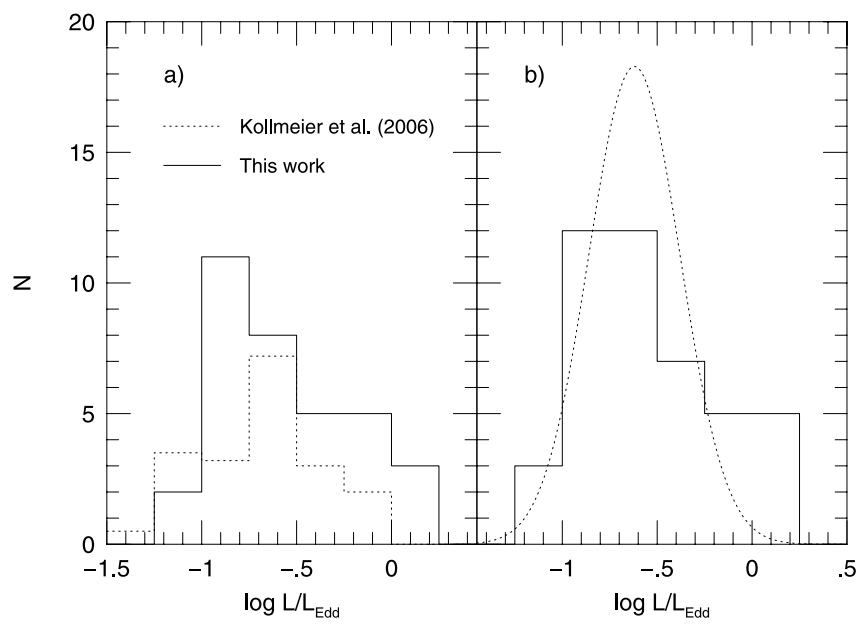

FIG. 3.- (a) Histograms of $L / L_{\text {Edd }}$ values as obtained from K06 (their Fig. 11 for $2<z<3$ sources with $M_{\mathrm{BH}}=10^{9}-10^{10} M_{\odot}$; dotted line) and this work (solid line; $M_{\mathrm{BH}}=10^{8.9}-10^{10} M_{\odot}$, using $f_{L}=7$ ). Note that we directly copied the data from the K06 paper; thus, the somewhat different bolometric correction used by those authors would shift their histogram to the left by about $0.1 \mathrm{dex}$. (b) A comparison of the $\log \left(L / L_{\mathrm{Edd}}\right)$ distribution in our sample with the favored K06 model (dashed line).

estimate, since the discrepancy is mostly due to the range in $L / L_{\text {Edd }}$, which is even larger when this method is used.

To illustrate the above points we show in Figure $3 a$ one of the K06 histograms (their Fig. 11, panel with $2<z<3$ and $M_{\mathrm{BH}}=$ $10^{9}-10^{10}$ ) alongside the 34 objects we observed within a similar mass and redshift range. A visual inspection shows the broader distribution of $L / L_{\text {Edd }}$ in our sample (note that the K06 distribution should be shifted to the left by about 0.1 dex due to the different bolometric correction used in their work).

A more quantitative test can be made by comparing our sample with the entire high-luminosity, high-redshift subsample of 131 objects from K06 (Table 1), despite the different ranges in $z$ and $M_{\mathrm{BH}}$. The overlap between the two is large, but the mean luminosity and $\mathrm{BH}$ mass in our sample is somewhat larger. According to $\mathrm{K} 06, \log \left(L / L_{\mathrm{Edd}}\right)$ for this group is well fitted with a normal distribution, which is centered at -0.52 and has a measured (model) standard deviation of $0.28(0.24)$. As explained, the mean should be shifted to -0.62 to allow for the somewhat larger bolometric correction used by K06. Our sample of 44 sources shows a similar mean $(-0.56)$ but a considerably larger scatter of $\sim 0.35$. It also shows a positive skewness of $\sim 0.47$, in comparison with the K06 value of -0.02 . This implies that sources with larger values of $L / L_{\text {Edd }}$ are more abundant in our sample. All these points are illustrated in Figure $3 b$, where we compare the histogram of our measured values of $L / L_{\mathrm{Edd}}$ with the favored K06 distribution for the 131 high- $z$, high- $L$ sources.

We attempted to verify the null hypothesis that the values of $L / L_{\text {Edd }}$ in our sample are indeed drawn from a lognormal distribution. A two-tailed Kolmogorov-Smirnov test over a large range of possible mean and standard deviation gives inconclusive results. The highest probability case has a mean $\log \left(L / L_{\mathrm{Edd}}\right)=$ -0.59 and $\sigma=0.37$. The $p$-value of this case is 0.92 (i.e., the probability of such a lognormal distribution in our sample is $92 \%)$. We also find that a K06 distribution $\left[\log \left(L / L_{\text {Edd }}\right)=\right.$ $-0.62 \pm 0.24$ given our bolometric correction] can be rejected at the $92 \%$ level. Finally, we tested the suggestion of a very large mean $L / L_{\text {Edd }}$, close to unity, in a sample like ours. A series of Kolmogorov-Smirnov tests show that a log-normal distribution of any width can be rejected at the $99 \%$ confidence level for all cases where the mean $\log \left(L / L_{\mathrm{Edd}}\right)$ is -0.25 or larger. As shown 
in $\S 3.2$, this is relevant for the comparison with $\mathrm{BH}$ evolution models.

To conclude, our sample of high-luminosity, high-redshift sources seems to be characterized by a similar mean but a somewhat broader distribution in $L / L_{\text {Edd }}$ compared with the similar properties subsamples in K06. Clearly, some of the differences may be related to the way we chose our sample, and in particular the fact that it is not a real flux limited sample. This prevents us from reaching firm conclusions about the source of the difference at this stage. However, the method used here is, in our opinion, the best for measuring $M_{\mathrm{BH}}$ and is preferable to the $\mathrm{K} 06$ method (see $\S 3.4$ ). Given all this, we suggest that the distributions of our 44 sources shown in Figure 3 represent the AGN population, over this redshift and $M_{\mathrm{BH}}$ range, in the best way. The large range in $L / L_{\text {Edd }}$ (a factor of $\sim 20$ ) is probably real and is likely to represent correctly the intrinsic properties of the AGN population analyzed here.

\subsection{Comparison with BH Evolution Models}

The cosmic evolution of BHs can be modeled by using large, recently observed AGN samples. This requires a combination of the X-ray and optical luminosity functions (LFs), since the first is more complete, yet the latter probes much deeper at high redshifts. Of the numerous papers discussing such models, we focus on the works of Marconi et al. (2004), Merloni (2004), Hopkins et al. (2006), and Volonteri et al. (2006).

Marconi et al. (2004) and Merloni (2004) address the growth rate of active BHs, assuming a constant accretion rate, $L / L_{\text {Edd }}=1$. Both papers suggest a faster growth rate of very massive BHs at earlier epochs in order to fit the observed LFs. The more recent studies of Hopkins et al. (2006) and Volonteri et al. (2006) include estimates of the typical $L / L_{\text {Edd }}$ required to match the redshift-dependent LFs. According to the modeling of Hopkins et al., the history of accretion and observed properties are not identical, since the low accretion rate phase is hardly observed due to obscuration. In particular, the large mass, very high luminosity AGNs are observable only close to their peak luminosity and accretion rate. This results in a narrow accretion rate range around $L / L_{\mathrm{Edd}}=1$, consistent with the Marconi et al. (2004) assumption. Volonteri et al. (2006) used a combination of the Hopkins et al. results with theoretical merger rates expected in cold dark matter scenarios. The accretion rate in their models is changing with redshift, with $0.3 \leq L / L_{\text {Edd }} \leq 1$ for $3 \leq z \leq 6$.

The typical uncertainty on the values of $L / L_{\text {Edd }}$ in our sample is a factor of $\sim 2$, and hence the mean value is not significantly different from the above predictions with their own large uncertainties. However, the trend seems to be different and points in a different direction. In particular, the theoretical requirement of $L / L_{\mathrm{Edd}} \simeq 1$ at high redshift and large $\mathrm{BH}$ mass is not seen in many of our sources. In fact, the range of $L / L_{\text {Edd }}$ covered by our very high luminosity AGNs, from about 0.07 to about 1.7 (with the Bentz et al. 2007 expression the range is 0.08-3.2), is not very different from the range typically observed in low-redshift AGN samples that are 2-3 orders of magnitude less luminous. Thus, it seems that some large-mass high-redshift active BHs are observed far from their peak luminosity phase, which presents a challenge to current theoretical models. Unfortunately, our sample does not go deep enough to search for even lower $L / L_{\text {Edd }}$ sources at high redshifts, and the K06 data does not help either, since all their $z>2$ mass measurements are based on the $\mathrm{C}$ IV $\lambda 1549$ method.

Finally, we mention several recent publications that attempt to measure mass and accretion rates in even higher redshift sources.
Jiang et al. (2006) presented Spitzer photometry of 13 AGNs at $z \sim 6$. The BH mass and accretion rate of four of the sources were estimated by the $\mathrm{C}$ IV $\lambda 1549$ method, and the $M_{\mathrm{BH}}$ and $L / L_{\text {Edd }}$ values are given in their Table 3 . All four BH masses are very large, $\sim 5 \times 10^{9} M_{\odot}$, and the $L / L_{\text {Edd }}$ range is $0.5-1$. However, the bolometric correction used by these authors is based on integrating over the entire spectrum, from hard X-rays to the farinfrared. This involves double counting, since almost half of this emission is due to reprocessing of the primary continuum (accretion disk and X-ray source) by the dusty medium near the $\mathrm{BH}$. Thus, the "true" $L / L_{\text {Edd }}$ values are smaller than those listed in their paper by a factor of $\sim 2$. Moreover, the BH mass estimates are rather uncertain due to the use of the $C$ IV $\lambda 1549$ method (see §3.4). Results of similar quality for a handful of additional AGNs at $z \sim 6$ have also appeared recently (e.g., Willott et al. 2003; Jiang et al. 2007; Kurk et al. 2007).

\subsection{The Growth Rate and Growth Time of High-Mass, High-Redshift BHs}

The combination of the newly measured BH masses and accretion rates can be used to estimate the growth times of massive BHs at $z \sim 2.3$ and 3.4. We follow the procedure outlined in NT07 (their eq. [6]) to calculate $t_{\text {grow }}$ assuming

$$
t_{\text {grow }}=t_{\text {Edd }} \frac{\eta /(1-\eta)}{f_{L} L_{5100} / L_{\text {Edd }}} \log \left(\frac{M_{\mathrm{BH}}}{M_{\text {seed }}}\right) \frac{1}{f_{\text {active }}},
$$

where $t_{\text {Edd }}=3.8 \times 10^{8} \mathrm{yr}$ for cosmic abundance, $\eta$ is the accretion efficiency, and $f_{\text {active }}$ is the duty cycle (the fractional activity time) of the BH. The growth time, $t_{\text {grow }}$, is most sensitive to $\eta$ and $f_{\text {active }}$ since both can vary by large factors. The seed BH mass, $M_{\text {seed }}$, may also change over a large range, but $t_{\text {grow }}$ is not very sensitive to this change. A small seed $\mathrm{BH}\left(10^{2}-10^{3} M_{\odot}\right)$ can be the result of Population III stars at $z \sim 20$, while larger seed BHs $\left(10^{4}-10^{6} M_{\odot}\right)$ can be due to direct collapse at lower redshifts (Begelman et al. 2006). Finally, $t_{\text {grow }}$ is less sensitive to $f_{L}$ because of the limited expected range of this factor in the sample at hand.

As discussed in several publications, most recently by King \& Pringle (2006), the value of $\eta$ depends on the direction and magnitude of the BH spin and the angular momentum of the accretion disk. The highest value is $\sim 0.4$ and the lowest values $(\sim 0.04)$ is obtained for retrograde accretion with a BH spin parameter of $a=-1$. More typical values, which reflect several spin-up and spin-down episodes with small-mass disks (see King \& Pringle 2006), give $\eta$ in the range of $0.05-0.08$. The values of $t_{\text {grow }}$ calculated here (Table 2) are for the case of $\eta=$ $0.1, M_{\text {seed }}=10^{4} M_{\odot}, f_{\text {active }}=1$, and $f_{L}=7$. We list them as $t_{\text {grow }} / t_{\text {universe }}$, where $t_{\text {universe }}$ is calculated at the given redshift of the source (using our adopted cosmology from $\S 2$ ).

The computed values of $t_{\text {grow }}$ exceed the age of the universe in most of the lower $L / L_{\text {Edd }}$ sources. While somewhat smaller values of $t_{\text {grow }} / t_{\text {universe }}$ are indeed likely, given the expected range in $\eta$, we consider the numbers given in Table 2 as conservative lower limits, since $f_{\text {active }}$ is likely to be much smaller than unity. For example, general galaxy and $\mathrm{BH}$ growth considerations predict $f_{\text {active }} \lesssim 0.1$. This can be estimated from number counts of AGNs and galaxies at large redshifts, from estimates of typical timescales for powerful starbursts, and from models of BH and galaxy evolution. Another estimate is obtained by assuming that the ratio of BH mass to the host galaxy mass is similar to the typical value observed in the local universe (about 1/700). This translates, for most objects in our sample, to a host mass of $10^{12} M_{\odot}$ or larger. To 
build up such a large stellar mass would require continuous star formation, from very early times, at a rate of $\sim 300 M_{\odot} \mathrm{yr}^{-1}$, or a much higher star formation rate, with a star formation duty cycle that is less than unity. Given the much faster growth of BHs by accretion, their duty cycles would be considerably shorter. While some very different scenarios cannot be excluded on the basis of current observations, they are definitely not in accord with present-day galaxy formation models. Antihierarchical $\mathrm{BH}$ growth models would give faster $\mathrm{BH}$ growth at high redshifts with a larger BH-to-galaxy mass ratio. It remains to be seen whether any such model is consistent with $f_{\text {active }} \sim 1$ all the way to redshift 3 for BHs like the ones in our sample.

Given our more plausible assumption of $f_{\text {active }} \ll 1$ for $z=$ 2-3 very large mass BHs, all 15 new sources presented here, and several of the remaining more luminous objects, require too much time to grow to their observed size. This problem was not noted in earlier works since most of them assumed $L / L_{\text {Edd }} \sim 1$, and hence $t_{\text {grow }}$ was an order of magnitude shorter. The problem is most severe for the lowest accretion rate sources. The higher accretion rate sources (most of the 29 objects from S04) have just enough time to explain their mass if $f_{\text {active }} \sim 1$. Thus, our new measurements, and general considerations, indicate that some very massive BHs had just enough time to grow to their measured size at redshifts $\sim 2.3$ and $\sim 3.4$. However, for all sources with $L / L_{\text {Edd }} \lesssim 0.3$, there was not enough time at redshift $\sim 3.4$, and even at redshift $\sim 2.3$, to grow to their observed size. This problem is more severe if we adopt the Hopkins et al. (2006) set of models, where much of the $\mathrm{BH}$ growth is during times when $L / L_{\mathrm{Edd}}$ is smaller than the time when the object is not obscured.

We conclude that a significant fraction of the sources in our sample must have had at least one previous episode of faster growth, probably with $L / L_{\text {Edd }} \sim 1$ and at $z \gtrsim 2-3$, in order to explain their BH mass. Given this, many extremely large mass BHs at redshifts 2 and 3 are in the process of their second and perhaps even third or fourth episode of activity. This does not necessarily apply for small-mass BHs at those redshifts that may have a different growth pattern.

\subsection{IV $\lambda 1549$ as a BH-Mass Indicator}

As noted above, the $\mathrm{C}$ IV $\lambda 1549$ method for estimating $\mathrm{BH}$ masses is problematic, since $C$ IV $\lambda 1549$ lines are known to show unusual profiles in many cases, including an enhanced blue wing and a large wavelength shift relative to the systemic velocity. This issue has been discussed, extensively, in numerous papers. The work of Baskin \& Laor (2005) and Sulentic et al. (2007) clearly show the differences between the $\mathrm{H} \beta$ and $\mathrm{C}$ IV $\lambda 1549$ line profiles and the problematics of FWHM(C IV $\lambda 1549)$ as a virial-motion indicator (see also the recent Shang et al. 2007 work). However, according to Vestergaard \& Peterson (2006), a proper calibration of FWHM(C IV 21549) and the UV continuum provides an adequate replacement for the $\mathrm{H} \beta$ method. K06 adopted this view and used the $\mathrm{C}$ IV $\lambda 1549$ method to estimate $\mathrm{BH}$ masses in many high-redshift sources.

Our combined sample includes 44 extremely luminous AGNs with $\mathrm{FWHM}(\mathrm{H} \beta)$ and $L_{5100}$ values for all. We have compiled $\lambda L_{\lambda}(1450 \AA)$ and FWHM(C IV $\left.\lambda 1549\right)$ for 42 of these and are thus in a position to compare the $\mathrm{H} \beta$ and $\mathrm{C}$ IV $\lambda 1549$ methods in the high-luminosity range. We first checked $L_{5100}$ versus $\lambda L_{\lambda}(1450 \AA)$ for all sources, where we have independent luminosities for both (i.e., excluding the six sources from S04 for which optical luminosities were estimated from their UV luminosities). We find a very significant low-scatter correlation, which is consistent with $L_{\nu} \propto \nu^{-0.5}$. The diagram (not shown here) sug-

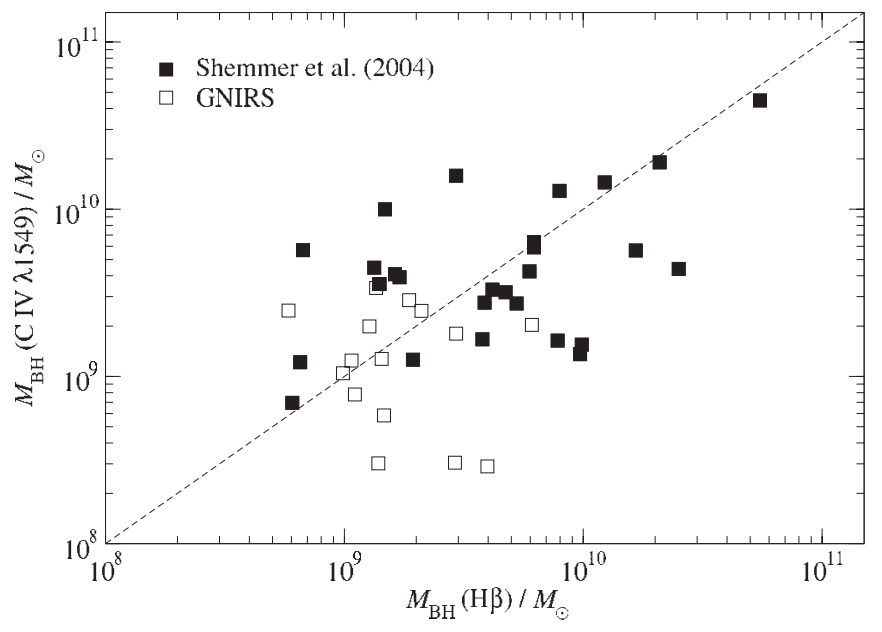

FIG. 4.- BH mass calculated with the $\mathrm{H} \beta$ method vs. the one obtained from the combination of FWHM(C IV 21549$)$ and the UV continuum. Symbols are the same as in Fig. 2. The dashed line marks a 1:1 correspondence to guide the eye.

gests that the two estimates of the source luminosity can be used interchangeably, with a different normalization, for estimating the BLR size. We then obtained BH masses by using the two methods: the $\mathrm{H} \beta$ method (eq. [1]) and the $\mathrm{C}$ IV $\lambda 1549$ method with the expression given in Vestergaard \& Peterson (2006). The two mass estimates are shown in Figure 4. The figure is a complete scatter diagram with no apparent correlation or trend. While the median (0.89) and the mean (1.49) $M_{\mathrm{BH}}(\mathrm{C}$ Iv 21549$) / M_{\mathrm{BH}}(\mathrm{H} \beta)$ ratio are not far from unity, the scatter is about \pm 0.3 dex.

Repeating the same analysis by using the Bentz et al. (2007) expression also gives a scatter diagram with no significant correlation. In this case the median and the mean for $M_{\mathrm{BH}}(\mathrm{C}$ IV $\lambda 1549) /$ $M_{\mathrm{BH}}(\mathrm{H} \beta)$ are 1.3 and 2.2, respectively, and the scatter is about 0.4 dex. This may provide a slight indication that the slope of $\alpha=0.65$ used in our work is preferable to the one used by Bentz et al. (2007), since, using this slope, the agreement between the median $M_{\mathrm{BH}}(\mathrm{C}$ IV 21549$)$ and the median $M_{\mathrm{BH}}(\mathrm{H} \beta)$ is improved.

The lack of correlation between $M_{\mathrm{BH}}(\mathrm{H} \beta)$ and $M_{\mathrm{BH}}(\mathrm{C}$ IV $\lambda 1549$ ) suggests that, while $\mathrm{BH}$ mass estimates based on the $\mathrm{C}$ IV $\lambda 1549$ line properties roughly follow the increase in source luminosity, their use is rather limited and highly uncertain, in the high-luminosity and/or high-redshift range. This conclusion is independent of the slope used to obtain $M_{\mathrm{BH}}(\mathrm{H} \beta)$. As argued earlier, some of the differences found between our $L / L_{\text {Edd }}$ distribution and the one presented in K06 are likely to be the result of their use of the C IV $\lambda 1549$ method. In fact, we notice a complete lack of correlation between the $L / L_{\text {Edd }}$ values derived with the $\mathrm{H} \beta$ method and those derived with the $\mathrm{C}$ IV $\lambda 1549$ method. Given all this, we suspect that the unexpected trend found by K06 when comparing the various line-based mass measurements (see their Fig. 4, and related discussion) is due to the problematic C IV $\lambda 1549$ method.

\section{CONCLUSIONS}

We have presented new, high-quality observations of the $\mathrm{H} \beta$ spectral region in 15 high-luminosity, high-redshift AGNs. We used the new data, in combination with our earlier observations of 29 sources, to compile a sample of 44 high-luminosity AGNs, where the $\mathrm{BH}$ mass estimates are all based on the $\mathrm{H} \beta$ method. The main findings are:

1. Our sample is the largest of its type and can therefore be used to investigate, in a more reliable way, the important correlations 
between source luminosity, BH mass, and accretion rate. Such measurements at high redshifts can be combined with similar measurements at $z<0.75$ to follow BH growth and AGN evolution through time.

2. There is a significant correlation between $L_{5100}$ and $L / L_{\mathrm{Edd}}$, but no correlation between $M_{\mathrm{BH}}$ and $L / L_{\mathrm{Edd}}$. These results do not depend on the exact slope used to derive $M_{\mathrm{BH}}$.

3. Assuming the distribution in $L / L_{\mathrm{Edd}}$ found here represents the high-redshift, high-luminosity AGN population, we find a significant fraction of sources with small $(\leq 0.2) L / L_{\text {Edd }}$. Current theoretical models predict only very few such sources among high-redshift AGNs.

4. Low accretion rate $\mathrm{BHs}$ represent a real challenge to $\mathrm{BH}$ growth scenarios. One possible way out is to assume that in all such cases, there was at least one earlier $(z>2.3$ or 3.4$)$ episode of $\mathrm{BH}$ growth with a higher accretion rate.

5. We present new evidence for the large uncertainty and probably systematic error associated with the use of the C IV $\lambda 1549$ method for estimating $M_{\mathrm{BH}}$ and hence $L / L_{\mathrm{Edd}}$ in high-redshift, highluminosity AGNs.

We thank J. M. Wang for useful discussions, Dovi Poznanski for assistance with the synthetic photometry, and an anonymous referee for useful suggestions. Funding for this work has been provided by the Israel Science Foundation grant 232/03 (H. N. and B. T.), by the Jack Adler chair of Extragalactic Astronomy at Tel Aviv University (H. N.), and by Project Fondecyt 1040719 (P. L.). This work is based on observations obtained at the Gemini Observatory, which is operated by the Association of Universities for Research in Astronomy, Inc. under a cooperative agreement with the NSF on behalf of the Gemini partnership: the National Science Foundation (United States), the Particle Physics and Astronomy Research Council (United Kingdom), the National Research Council (Canada), CONICYT (Chile), the Australian Research Council (Australia), CNPq (Brazil), and CONICET (Argentina).
Baskin, A., \& Laor, A. 2005, MNRAS, 356, 1029

Begelman, M. C., Volonteri, M., \& Rees, M. J. 2006, MNRAS, 370, 289

Bentz, M. C., Denney, K. Y., Peterson, B. M., \& Pogge, R. 2007, in ASP Conf. Ser. 373, The Central Engine of Active Galactic Nuclei, ed. L. C. Ho \& J.-M. Wang (San Francisco: ASP)

Bentz, M. C., Peterson, B. M., Pogge, R. W., Vestergaard, M., \& Onken, C. A. 2006, ApJ, 644, 133

Boroson, T. A., \& Green, R. F. 1992, ApJS, 80, 109

Cutri, R. M., et al. 2003, The IRSA 2MASS All Sky Catalog of Point Sources (Pasadena: NASA/IPAC)

Hopkins, P. F., Hernquist, L., Cox, T. J., Di Matteo, T., Robertson, B., \& Springel, V. 2006, ApJS, 163, 1

Jiang, L., Fan, X., Vestergaard, M., Kurk, J. D., Walter, F., Kelly, B. C., \& Strauss, M. A. 2007, AJ, 134, 1150

Jiang, L., et al. 2006, AJ, 132, 2127

Kaspi, S., Brandt, W. N., Maoz, D., Netzer, H., Schneider, D. P., \& Shemmer, O. 2007, ApJ, 659, 997

Kaspi, S., Maoz, D., Netzer, H., Peterson, B. M., Vestergaard, M., \& Jannuzi, B. T. 2005, ApJ, 629, 61 (K05)

Kaspi, S., Smith, P. S., Netzer, H., Maoz, D., Jannuzi, B. T., \& Giveon, U. 2000, ApJ, 533, 631

King, A. R., \& Pringle, J. E. 2006, MNRAS, 373, L90
REFERENCES

Kollmeier, J. A., et al. 2006, ApJ, 648, 128 (K06)

Kurk, J. D., et al. 2007, ApJ, 669, 32

Marconi, A., Risaliti, G., Gilli, R., Hunt, L. K., Maiolino, R., \& Salvati, M. 2004, MNRAS, 351, 169

McLure, R. J., \& Dunlop, J. S. 2004, MNRAS, 352, 1390

Merloni, A. 2004, MNRAS, 353, 1035

Netzer, H., Shemmer, O., Maiolino, R., Oliva, E., Croom, S., Corbett, E., \& di Fabrizio, L. 2004, ApJ, 614, 558

Netzer, H., \& Trakhtenbrot, B. 2007, ApJ, 654, 754 (NT07)

Richards, G. T., et al. 2006, ApJS, 166, 470

Shang, Z., Wills, B. J., Wills, D., \& Brotherton, M. S. 2007, AJ, 134, 294

Shemmer, O., Netzer, H., Maiolino, R., Oliva, E., Croom, S., Corbett, E., \& di Fabrizio, L. 2004, ApJ, 614, 547 (S04)

Sulentic, J. W., Bacher, R., Marziani, P., Negrete, C. A., \& Dultzin, D. 2007, ApJ, 666, 757

Sulentic, J. W., Repetto, P., Stirpe, G. M., Marziani, P., Dultzin-Hacyan, D., \& Calvani, M. 2006, A\&A, 456, 929

Vestergaard, M. 2004, ApJ, 601, 676

Vestergaard, M., \& Peterson, B. M. 2006, ApJ, 641, 689

Volonteri, M., Salvaterra, R., \& Haardt, F. 2006, MNRAS, 373, 121

Willott, C. J., McLure, R. J., \& Jarvis, M. J. 2003, ApJ, 587, L15

York, D. G., et al. 2000, AJ, 120, 1579 